\title{
Ueber Primzahlen in aufeinander folgenden Intervallen.
}

\author{
Von Richard Obláth (in Budapest).
}

In früheren Publikationen (') habe ich den Satz mitgeteilt:

Bei hinreichend grossem $\mathrm{x}$ enthält jedes der gleich langen Intervalle

$$
\langle 1, \ldots, x\rangle,\langle x, \ldots, 2 x\rangle, \ldots,\langle(n-1) x, \ldots, n x\rangle
$$

weniger Primzahlen als das Vorhergehende, wenn

$$
3 n<\log x
$$

ist.

Die Herren Michael Bauer und Werner Weber hatten die Liebens. würdigkeit, mich aufmerksam zu machen, dass mein Beweis diese Aussage nicht vollständig deckt, und zwar hauptsächlich deshalb, weil die benützte Form des Primzahlsatzes nicht genug scharf war. Der Satz selber ist jedoch richtig, man kann sogar wesentlich mehr behaupten. Es gilt nämlich der folgende:

SATz 1. - Bei hinreichend grossem $\mathrm{x}$ enthölt jedes der Tutervalle (1) von der Länge $\mathbf{x}$ weniger Primadhlen als das Vorhergehende, solange

$$
n<\log ^{m} x
$$

ist, wo m eine beliebige feste ganze Zahl bedeutet.

Dieser Satz, den ich einer freundlichen Mitteilung des Herren PAuL ERDös verdanke, besagt deshalb mehr als der Vorausgeschickte, weil infolge der letzten Ungleichung, $n$ - die Anzahl derjenigen Intervalle, in welchen die monotone Abnahme der Primzahlhänfigkeit sichergestellt ist - jede Potenz von $\log x$ überschreiten darf, während nach (2) $n$ nur von der Grössenordnung des $\log x$ ist. Herr WEBER teilte mir einen Beweis mit, nach welchem $m \leqq 9$ ansfallen kann.

(1) OpLÀre, "Math. és. Termtud. Eirt. ", 47, (1980), p. 250; und "Tờ. Math. Journ.", 32 , (1930), p. 328 . 
Der Beweis beruht auf dem Primzahlsatze mit dem von Herren LiT. TLEWroon gegebenen Restgliede $\left(^{2}\right)$, nach welchem die Anzahl der $x$ nicht übertreffenden Primzahlen

$$
\pi(x)=\int_{3}^{x} \frac{d z}{\log z}+O\left(x e^{-\alpha \mid \log x \log \log x}\right)
$$

beträgt, wo $\alpha$ eine positive Konstante bezeichnet, auf deren numerischen Wert es nicht ankommt. Die Anzahl der Primzahlen zwischen $(k-1) x$ und $k x$ liefert daher die Formel

$$
\begin{gathered}
u_{k}(x)=\pi(k x)-\pi((k-1) x)= \\
=\int_{(k-1) x}^{k x} \frac{d \tilde{z}}{\log z}+O\left(k x e^{-x \sqrt{\log (k-1) x \log \log (k-1) x}) .}\right.
\end{gathered}
$$

Die positive ganze Zahl $k$ haben wir so zu wählen, dass für $k<n$

zutreffen soll.

$$
u_{k+1}(x)<u_{k}(x)
$$

Aus der Formel (4) folgt

$$
\begin{gathered}
U_{k}(x)=u_{k}(x)-u_{k+1}(x)= \\
=\int_{(k-1) x}^{k x} \frac{d z}{\log z}-\int_{k x x}^{(k+1) x} \frac{d z}{\log z}+O\left(k x e^{-x+\overline{\log x} \overline{\log \log x}}\right)= \\
=\int_{k=1}^{k x}\left(\frac{1}{\log z}-\frac{1}{\log (z+x)}\right) d z+O\left(k x e^{-z \sqrt{\log x \log \log x}}\right)
\end{gathered}
$$

wobei das Ordoglied vergrössert wurde. Der Integrand nimmt für $z \geqq 2$ monoton ab, wovon man durch Differenziieren iberzeugt wird $\left({ }^{5}\right)$. Wenn daher

(2) Landau, "Math. Zeitschrft. ", 20, (1924), p. 90, besonders p. 103, und ebenda p. 105. S. weiter Landad, Zahlentheorie II, (1927), p. 47, Satz 403. Meines Wissens ist Hermn LitTLwoods Annaherung noch immer die beste. S. z. B. INGHAM, The Distr. of Prime Numbers. ("Cambridge Tracts in Math. n. ${ }^{\circ} 30$ "), Cambridge, 1932, p. 66, Theor. 24.

(3) In meinen zitierten Arbeiten habe ich den Satz schon für jedes $k<M$ bewiesen, wo $M$ eine beliebige gegebene Zahl bèdentet; der Nachweis der Tatsache $u_{2}(x)<u_{1}(x)$ stammt schon von Herrn Landau "Nouv. Ann. de Math." s. 4, t. 1, p. 281, (1901). Wir brauchen uns also nur um grosse $k-\mathrm{s}$ zu kimmern. Die für $k=1$ nötige Modifikation lässt sich aber auch aus der hier benïtzten schärferen Formel in wenigen Strichen entwickeln, 
für das in $U_{k}(x)$ auftretende Integral ohne Restglied $V_{k}(x)$ gesetzt wird, dann hat man (') für $k \geqq 2$

$$
\begin{aligned}
& U_{k}(x)=\int_{(k-1) x}^{k x}\left(\frac{1}{\log z}-\frac{1}{\log (z+x)}\right) d z \geqq x\left(\frac{1}{\log k x}-\frac{1}{\log (k+1) x}\right)= \\
& =\frac{x \log \left(1+\frac{1}{k}\right)}{\log k x \log (k+1) x}>x \frac{\log \left(1+\frac{1}{k+1}\right)}{\log ^{2}(k+1) x}>\frac{\gamma x}{(k+1) \log ^{2}}(k+1) x
\end{aligned}
$$

wo $\gamma$ eine positive absolute Konstante bezeichnet. Nach der Fussnote ( $\left.{ }^{3}\right)$ gilt diese Abschätzung auch für $k=1$.

Der Unterschied der Anzahlen der Primzahlen in beiden benachbarten Intervallen beträgt also

$$
U_{k}(x) \geqq\left(\frac{\gamma x}{(k+1) \log ^{2}(k+1) x}+O\left(k x e^{-x} \cdot \overline{\log x \log \log x}\right) .\right.
$$

Damit es im letzteren Intervalle weniger Primzahlen gebe als im Vorher: gehenden, ist das Ueberwiegen des ersten Gliedes hinreichend, d. h. es muss

$$
\frac{\gamma x}{(k+1) \log ^{2}(k+1) x}>O\left(k x e^{-a+1 \log x \log \log x}\right)
$$

stattfinden. Dieser Bedingnng kann durch

$$
e^{x \sqrt{\log x \log \log x}}>0\left((k+1)^{2} \log ^{2}(k+1) x\right)
$$

denn es ist

$$
\begin{gathered}
V_{1}(x)=\int_{2}^{x} \log z-\int_{x}^{23 x} \frac{d z}{\log z}=\int_{z}^{x}\left(\frac{1}{\log z}-\frac{1}{\log (z+x)}\right) d z-\int_{x}^{x+2} \frac{d z}{\log z} \geq \\
\geq(x-2)\left(\frac{1}{\log x}-\frac{1}{\log 2 x}\right)-\frac{2}{\log x}
\end{gathered}
$$

daher lat man für cin hinreichend grosses $x$

$$
V_{1}(x)>\beta x\left(\frac{1}{\log x}-\frac{1}{\log 2 x}\right)
$$

wo $\$$ eine positive absolute Konstante bezeichnet.

(i) Weil aus

$$
\begin{aligned}
& \log \left(1+\frac{1}{k+1}\right)=\frac{1}{k+1}-\frac{1}{2}\left(\frac{1}{k+1}\right)^{2}+\frac{1}{3}\left(\frac{1}{k+1}\right)^{3}-+\ldots \\
& \log \left(1+\frac{1}{k+1}\right) \geq \frac{1}{k+1}\left(1-\frac{1}{2} \frac{1}{k+1}\right) \geq \begin{array}{l}
3 \\
1 k+1
\end{array}
\end{aligned}
$$

folgt. 
gentagt werden. Wenn also $k<x$ ist, finden wir die hinreichende Bedingung

$$
e^{x} \overline{\log x \log \log x}>O\left(k^{2} \log ^{2} x\right) .
$$

Die Anzahl der in den Intervallen der Folge (1) enthaltenen Primzahlen nimmt daher bei der Wahl

$$
k=o\left(\frac{e^{\frac{x}{2} \cdot \overline{\log x \log \log x}}}{\log x}\right)
$$

monoton ab. Eine einfache Rechnung zeigt, dass - für $k$ seinen grössten zulassigen Wert $n$ eingesetzt - die Bedingung (6) durch die Wahl

$$
n<\log ^{m n} x
$$

befriedigt wird, wo $m$ eine beliebige positive ganze Zahl sein darf, womit unser Satz vollständig bewiesen ist.

Ein analoger Satz besteht für die Primzahlen der arithnetischen Reihe.

Unser Satz gibt eine Folge von Intervallen an, in welchen die Prim. zahlhäufigkeit monoton abnimmt. Als Gegenstuck dazu beweisen wir den:

SATZ 2. - Es sei $\lambda>1$ eine positive Konstante. Für ein hinreichend grosses $\mathrm{x}$ enthält jedes der Intervalle

$$
<x, \ldots, \lambda x\rangle,\left\langle\lambda x, \ldots, \lambda^{2} x>, \ldots,<\lambda^{n-1} x, \ldots, \lambda^{n} x>, \ldots\right.
$$

mehr Primzahlen als das Vorhergehende.

Der Beweis ist dem Vorangegangenen nachgebidet.

Die Differenz der Anzahl der in zwei benachbarten Intervallen befindlichen Primzahlen ist also (")

$$
\begin{gathered}
U_{n}(x)=\int_{\lambda^{n} x}^{\log ^{n+1} x}-\int_{\lambda^{n+1} x}^{\lambda^{n} x} \frac{d z}{\log z}+O\left(\lambda^{n+1} x e^{-\alpha \sqrt{\log \lambda^{n} x} \log \log \lambda^{n} x}\right)= \\
\left.=\int_{\lambda^{n} x}^{\lambda^{n+1} x} \frac{d z}{\log z} \frac{1}{\lambda} \frac{d z}{\log \frac{z}{\lambda}}\right)+O\left(\lambda^{n+1} x e^{-\alpha \sqrt{\log \lambda^{n} x \log \log \lambda^{n} x}}\right) .
\end{gathered}
$$

(5) Bei der Bewertung des Ordogliedes denke man daran, dass zu grösseren Werten der Variablen ein grösseres Ordoglied gehört, es ist also

$$
O\left(\lambda^{n+1} x e^{-x} \sqrt{\log \lambda^{n+1} x \log \log \lambda^{n+1} x}\right) \leqq O\left(\lambda^{n+1} x e^{-\alpha \cdot \sqrt{\log \lambda^{n} x \log \log \lambda^{n} x}}\right) .
$$

Das Restglied des Textes ist daher richtig. 
Der Integrand nimmt fuir $z>\lambda^{\frac{\sqrt{\bar{\lambda}}}{\sqrt{\bar{\lambda}-1}}}$ monoton ab. Wenn also das Integral ohne Restglied wieder mit $V_{n}(x)$ bezeichnet wird, erhalten wir die Abschätzung

$$
V_{n}(x)=\int_{\lambda^{n} x}^{\lambda^{n+1} x}\left(\frac{d z}{\log z}-\frac{d z}{\lambda \log \frac{z}{\lambda}}\right)>\left(\lambda^{n+1} x-\lambda^{n} x\right)\left(\frac{1}{\log \lambda^{n+1} x}-\frac{1}{\lambda \log \lambda^{n} x}\right) .
$$

Wir setzen $\lambda^{n} x=y$ und schreiben

$$
V_{n}(x)>y(\lambda-1)\left(\frac{1}{\log \lambda y}-\frac{1}{\lambda \log y}\right)
$$

Der Ausdruck rechts ubbertrifft stets das Ordoglied wegen

$$
\begin{aligned}
& (\lambda-1) \frac{\lambda \log y-\log \lambda y}{\log y \log \lambda y} e^{x / \overline{\log y \log \log y}}> \\
& >(\lambda-1) \frac{\lambda \log y-\log \lambda y}{\log y \log \lambda y} e^{\log \left(\log ^{n z} y\right)}> \\
& >(\lambda-1) \frac{\lambda \log y-\log \lambda y}{\log y \log \lambda y} \log m y>\log ^{\nu} y
\end{aligned}
$$

wo $m$ eine beliebige ganze Zahl bedeutet, und $\vee<m$ ist. Q. e. $d$.

Ein analoger Satz besteht für die Primzahlen der arithmetischen Reihe.

Ich erfülle eine angenehme Pflicht, indem ich den Herren Mrorate Bauer und Paul ERdös, deren wertvolle Bemerkungen ich auch im Beweise verwenden konnte, meinen herzlichsten Dank ausspreche. 